\title{
A metabolic study in hepatopancreas of Litopenaeus vannamei response to white spot syndrome virus
}

\author{
Hao Wu 1 • Jianlong Zhang $\cdot$ Yongxing He $\cdot$ Junfang Zhou • \\ Jizhou Yan $\cdot$ Min Jiang
}

Received: 8 November 2016/Accepted: 19 April 2017/Published online: 17 May 2017

(C) The Author(s) 2017. This article is an open access publication

\begin{abstract}
White spot syndrome virus (WSSV) cause great harm in shrimp aquaculture. To understand the impact of viral infection on the shrimp metabolism, we monitored the culture farms of Litopenaeus vannamei and collected the samples on different stages of WSSV infection. The hepatopancreas of shrimp were separated, and then used gas chromatography mass spectrometry to detect the metabolites. Through the mass spectrometric analysis combined with multivariate data analysis, including PCA and OPLS models, metabolism of the shrimp was significantly changed by WSSV infection. The data indicated that in the early stage of WSSV infection, the glycolysis changed significantly, the contents of glucose and lactate increased distinctly. The metabolites of TCA cycle did not show up obviously regularity. The organism of fatty acids showed the same situation with glycolysis. At the early stage of infection, 14 amino acids metabolism were up-regulated, and glycine still increased at later stage of infection and the concentration was increased twice. The data of this study may provide some information to further research of viral disease mechanism.
\end{abstract}

Keywords Metabolomics · White spot syndrome virus · GCMS

\section{Introduction}

White spot syndrome virus (WSSV) often brought disastrous to shrimp farming industry. The virus was reported to infect most aquatic animals including crabs and shrimp (Lo et al. 1996; Sánchez-Paz 2010). So, it is very important to learn the mechanisms of virus attacks the host and the shrimp response to WSSV

Hao $\mathrm{Wu}$ and Jianlong Zhang have contributed equally to this work.

\footnotetext{
H. Wu

Key Laboratory of Freshwater Aquatic Genetic Resources, Ministry of Agriculture, Shanghai Ocean University, Shanghai 201306, China

H. Wu $\cdot$ J. Zhang $\cdot$ J. Yan $\cdot$ M. Jiang ( $₫)$

College of Fisheries \& Life Science, Shanghai Ocean Universities, Shanghai 201306, China

e-mail: mjiang@shou.edu.cn

Y. He

Agricultural Service Center of Pudong New Area, Shanghai, China

J. Zhou

Key and Open Laboratory of Marine and Estuarine Fisheries, Ministry of Agriculture, East China Sea Fisheries Research Institute, Chinese Academy of Fishery Sciences, Shanghai, China
} 
infection. Hepatopancrea was an important organ of shrimp metabolize. Some research had focused on genes, biochemical and physiological changes related to WSSV infection in shrimpand differentially expressed proteins in the hepatopancrea of shrimp (Yoganandhan 2003; Leu et al. 2007, 2013; Li et al. 2013) and upregulation and down-regulation proteins were identified. The above studies show that the host's metabolism will be affected after the WSSV virus invades, and this negative effect will lead to the shrimp's immune capacity decline or lead to death. To understand the reaction mechanism of WSSV infection, metabolism data were important supplement to the research. GCMS is a suitable instrumental to detective metabolites, which provides a rapid, steady, and high-throughput methodology. In this study, GCMS technique and multivariate statistics were applied to profile the hepatopancreas and approached for metabolic marker when Litopenaeus vannamei were infected by WSSV.

\section{Materials and methods}

Sample collection and treatment

There were several shrimp farms under our track, the aquaculture farm in this paper have 15 shrimp pounds. The shrimps would feed 4 or 5 times a day with shrimp fodder. And we monitor the Shrimp of every two weeks contain white spot virus tested and water quality monitored throughout the growing season. When the white spot virus was detected on the shrimp, the testing organisms with the virus and healthy control shrimp would be collected immediately and synchronous, samples were stored at $-80{ }^{\circ} \mathrm{C}$ ultra-low temperature freezer. The number of samples collected per time is greater than 25 , three whole shrimps for virus detection, and 20 shrimps for nutritional analysis and metabolic analysis with different body parts. We got three groups under same track time which contain healthy shrimp without virus as control (group-1), infected virus within 2 weeks as latent infection (group-2) and infected with the virus that causes floating and imminent death which is dominant infection (group-3). The weight range of all the samples is $1.97 \pm 0.66 \mathrm{~g}$. Quantitative detection of WSSV by RT-PCR. The hepatopancreas of shrimp would be homogenized in tube after weighted, then $250 \mu \mathrm{L}$ water-methanol-chloroform $(2: 5: 2 \mathrm{v} / \mathrm{v} / \mathrm{v})$ were mixed for metabolite extraction, and then kept at $-18{ }^{\circ} \mathrm{C}$ for $2 \mathrm{~h}$. After centrifugation at $10000 \mathrm{rpm}$ for $30 \mathrm{~min}, 100 \mu \mathrm{L}$ supernatant was dried completely, $80 \mu \mathrm{L}$ nitrogen-methoxyamine $\left(15 \mathrm{mg} \cdot \mathrm{mL}^{-1}\right.$ in pyridine) (Sigma-Aldrich, USA) and $80 \mu \mathrm{L}$ BSTFA (with $1 \%$ TMCS) (SUPELCO, USA)were added to the residue to trimethylsilylated at $60{ }^{\circ} \mathrm{C}$ for $60 \mathrm{~min}$.

Samples were analyzed by GCMS (7890A + 5975C, Agilent, USA) in non-target detection with a DB$17 \mathrm{MS}$ capillary column (J\&W Scientific, USA). $1 \mu \mathrm{L}$ of sample was injected in splitless mode. The initial temperature was kept at $50{ }^{\circ} \mathrm{C}$ for $2 \mathrm{~min}$, then raised with four steps, $100{ }^{\circ} \mathrm{C}$ at a rate of $8{ }^{\circ} \mathrm{C} / \mathrm{min}, 170{ }^{\circ} \mathrm{C}$ at a rate of $10{ }^{\circ} \mathrm{C} / \mathrm{min}$ and $200{ }^{\circ} \mathrm{C}$ at a rate of $5{ }^{\circ} \mathrm{C} / \mathrm{min}$, finally to $300{ }^{\circ} \mathrm{C}$ at a rate of $8{ }^{\circ} \mathrm{C} / \mathrm{min}$ and kept for $4 \mathrm{~min}$. The injection and ion source temperatures were 260 and $230{ }^{\circ} \mathrm{C}$, the energy was $70 \mathrm{eV}$ in electron impact mode. The mass spectrometry data were acquired in full-scan mode with the $\mathrm{m} / \mathrm{z}$ range of 55-600.

Data analyses

The acquired dataset in MS files from GCMS analysis was exported into NetCDF format by MSD ChemStation (Agilent, USA). CDF files were processed using XCMS-online (XCMS, USA). The soft would finish peaks of denoising, smoothing, time-window splitting, multivariate curve resolution and alignment. The results of three-dimensional dataset comprised sample information, peak retention time and intensity. After principal component analysis (PCA) analysis with SIMCA-14 (Umetrics, Sweden) and Metaboanalyst online analysis software (www.metaboanalyst.ca/), projection to latent structures discriminate analysis (PLS-DA) and orthogonal projection to latent structures discriminate analysis (OPLS-DA) were carried out to further analysis. The software SIMCA14 was used to try to identify marker metabolites from three groups. Metabolite identification was determined according to National Institute of Standards and Technology (NIST) spectral library databases. The software of SPSS20 was application to make significant difference analysis and charts. Quantitative normalization within replicates was transformed by logarithmic base of 2 (Xia and Wishart 2011; Wu et al. 2013). Using SPSS 20.0 software for ANOVA analysis of the metabolites' relative content between three groups. 


\section{Results}

\section{Multivariate analysis}

With the multivariate analysis of GCMS data, the score plot (Fig. $1, R^{2} X=98.6 \%, Q^{2}=0.941$ ) of PCA was obtained with the first three PCs which represented 49 and $17 \%$ variance, respectively. Then the POLS-DA model was used, the quality of model described by the cross-validation parameters $Q^{2}$, and the $R^{2} X$ values show the total number of the variation in the $X$ matrix, and the score plots showed evident distinction between three groups.

Differences in the detected metabolite between 3 groups

The results showed different levels of metabolism including glycolysis, TCA cycle, fatty acid and amino acid. The results showed increase in hepatopancreas glucose and lactate concentration in group 2 compared with
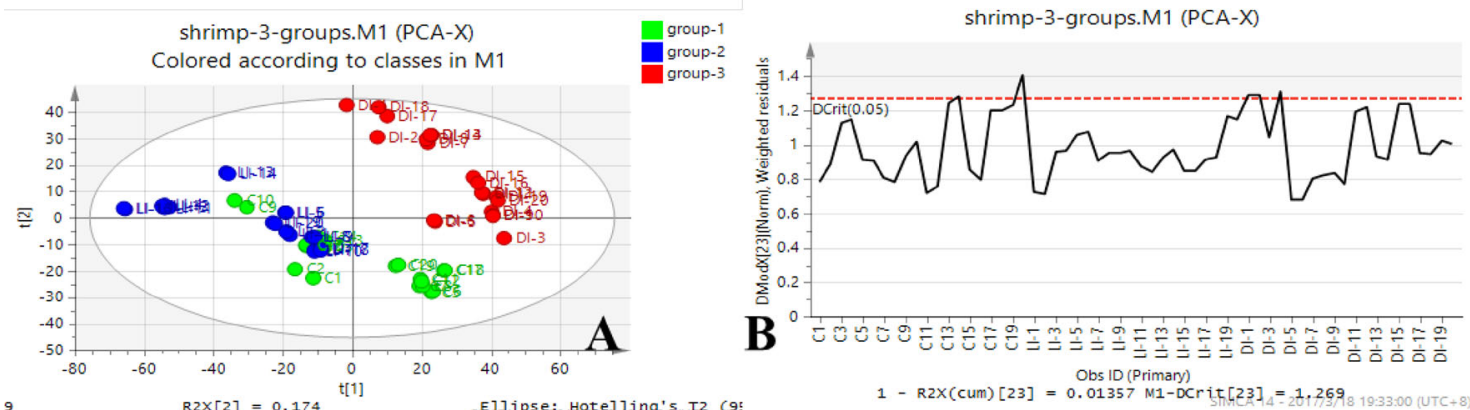
ETCAPSe: Hotelling's T2 69 !

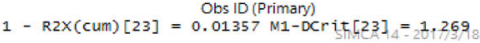
shrimp-3-groups.M2 (OPLS-DA)
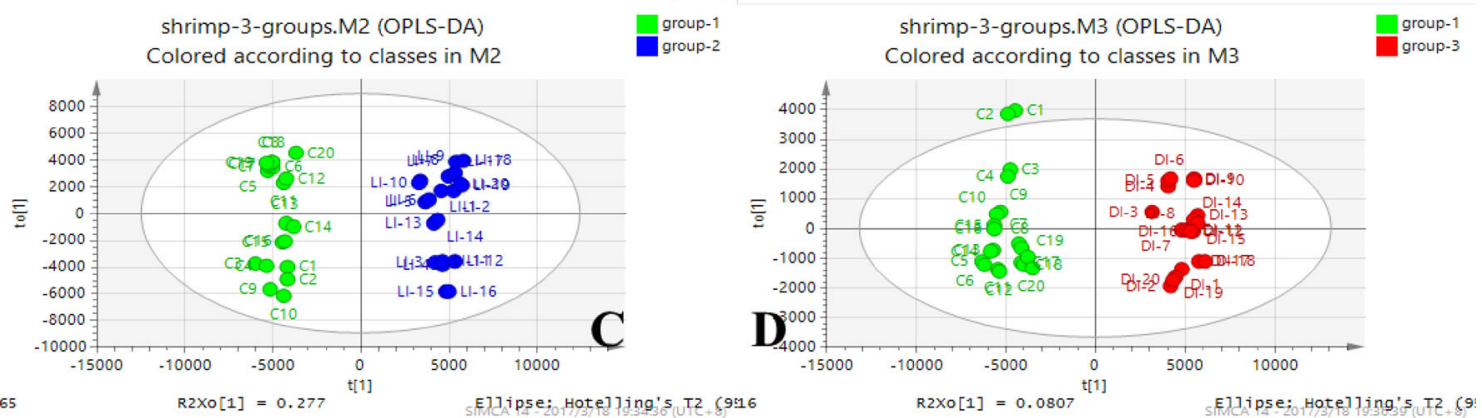

shrimp-3-groups.M2 (OPLS-DA): Validate Model 믈 $\mathrm{Q} 2$ \$M2.DA(group-1) Intercepts: $\mathrm{R} 2=(0.0,0.364), \mathrm{Q} 2=(0.0,-0.814) \quad \square \mathrm{Q} 2$
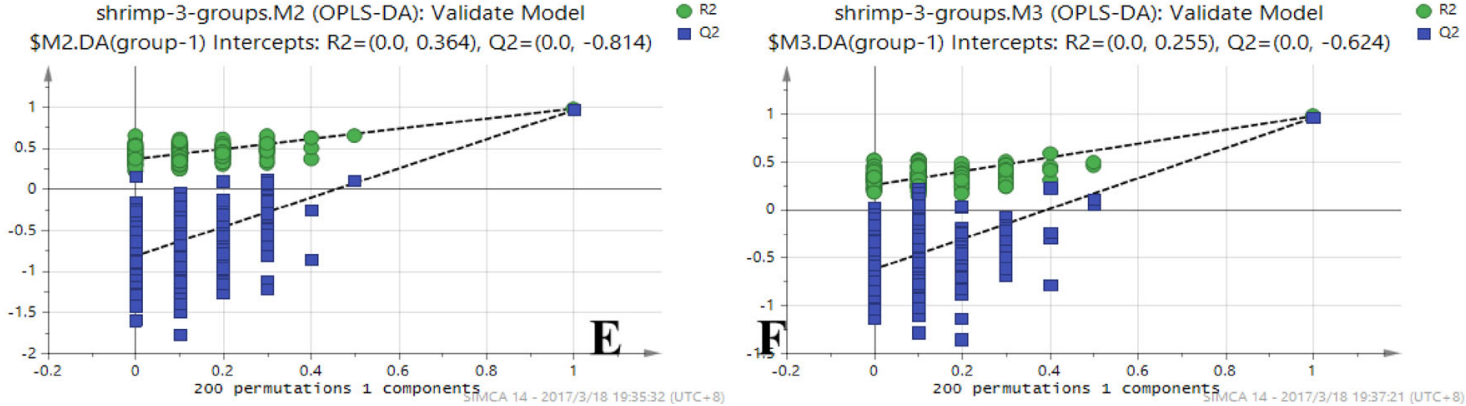

Fig. 1 There were significant differences in metabolomics levels between the three experimental groups by principal component analysis. a PCA scores plot on GCMS of shrimp hepatopancreas obtained from 3 groups. Green dot control (group-1); Blue dot WSSV latent infection stage (group-2); Red dot WSSV dominant infection stage (group-3). b A moderate outlier of 60 samples. c The scatter plot of the scores indicate that group control and latent infection group (group-2) can be a good distinction under the OPLS-DA model. d The scatter plot of the scores indicate that group control and dominant infection group (group-3) can be a good distinction under the OPLS-DA model. e The separation model was tested by permutation test, and the results showed that the model (c) between the control and LI groups met the requirements. $\mathbf{f}$ The separation model was tested by permutation test, and the results showed that the model (d) between the control and DI groups met the requirements 
control, but subsequently decrease in group 3 which the shrimp were WSSV dominant infection and immediately to death. The concentrations of pyruvate have no significant difference between three groups (Fig. 2). In the metabolic processes of the TCA cycle,the content of oxaloacetate from group 2 showed a significant $(p<0.05)$ increase, contrary succinate and $\alpha$-ketoglutarate showed a significant decrease. The concentration of fumarate and oxaloacetate changed higher than control, while succinate, malate and $\alpha$-ketoglutarate were decreased (Fig. 3). Fatty acid metabolism was similar with glycolysis, the concentration of detected fatty acids including palmitic acid, palmitoleic acid, stearic acid, linoleic acid, $\alpha$-linoleic acid and EPA, changed higher on group 2, and reduced on group 3 except stearic acid which still increased on group 3. Oleic acid did not significant increase on group 2, but changed higher on group 3 significantly (Fig. 4). Amino acid of serine and glutamine in three groups had no evidence difference. The WSSV infected shrimp had significantly higher contents of 14 amino acids on group 2, these metabolites including glycine, alanine, asparagine, valine, threonine, lysine, histidine, tryptophan, leucine, isoleucine, cysteine, arginine, phenylalanine and tyrosine. There were six amino acids changes in the concentration of group 3, the glycine and aspartic acid were upregulation and the contents of alanine; asparagine, proline and glutamate were lower than control (Table 1).

\section{Discussion}

To interact with their host cells, viruses have established a dynamic and intricate biological mechanism. They defuse the host defenses and induce extensive changes to cell metabolism (Bruggeman 2007; Heaton and Randall 2011). The results were similar when white spot syndrome virus infected the L. vannamei in this study; the results indicated that the WSSV-infected shrimps have a changed hepatopancreas metabolite profile. Compared with healthy controls, glucose of infected shrimp was increased but decreased at the time when the infected shrimp approaching to death. These results of this paper were like some previous research which described a significant increase in plasma glucose concentration of WSSV infected shrimp; they suggested that shrimp infected with virus may need to use glucose as an energy source to maintain of immune response to resist this rapidly replicating virus (Yoganandhan 2003; Galvan-Alvarez et al. 2012). Another research indicates that glucose and lactate concentration increased in WSSV-infected shrimp at the stage of viral genome replication, and the glucose-6-phosphate dehydrogenase showed increased activity which is crucial enzyme of pentose phosphate pathway and lactate was shuttled into gluconeogenesis under normal biochemical situation. The decrease trend of lactate indicated that capacity of hydrogen had been changed and it contributed to the gluconeogenic supply of glucose for energy needing the infected shrimps (Solanky et al. 2003; Chen et al. 2011; Liu et al. 2015). The time of group 2 shrimp which infected by WSSV were less than

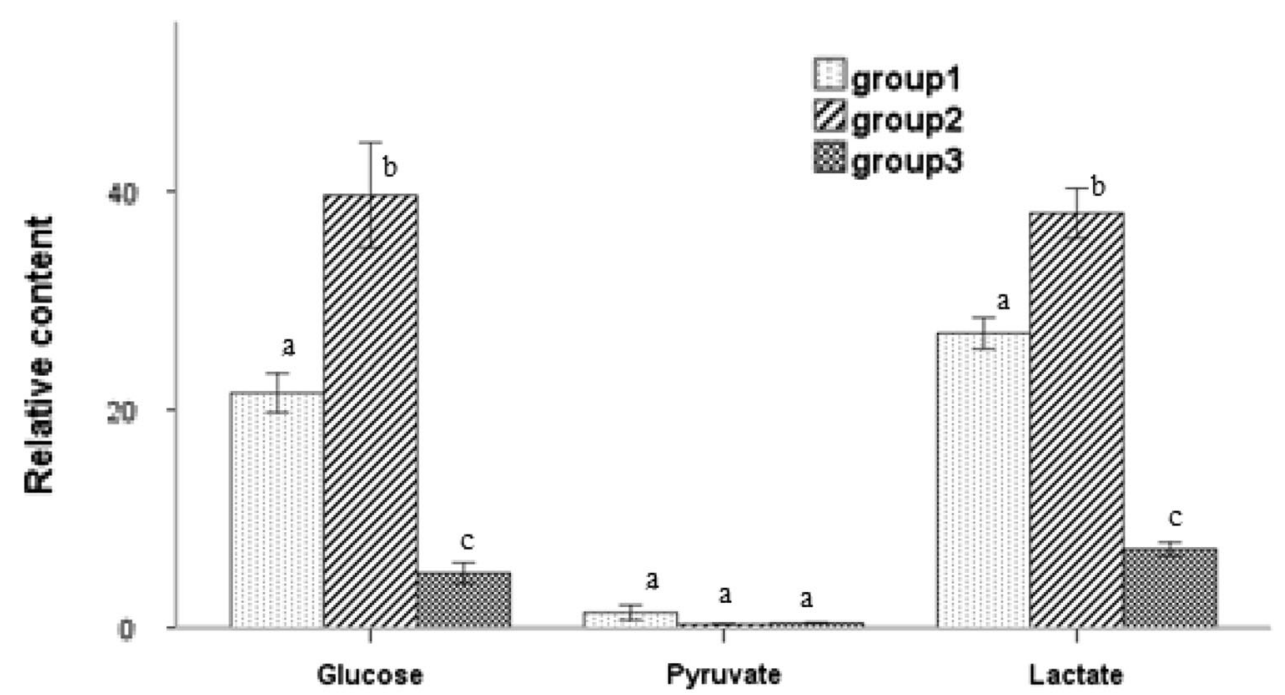

Fig. 2 Relative concentration of glycolysis metabolites in shrimp hepatopancreas after WSSV infected. " $a$ ", " $b$ ", " $c$ " indicate significant $(p<0.05)$ difference 


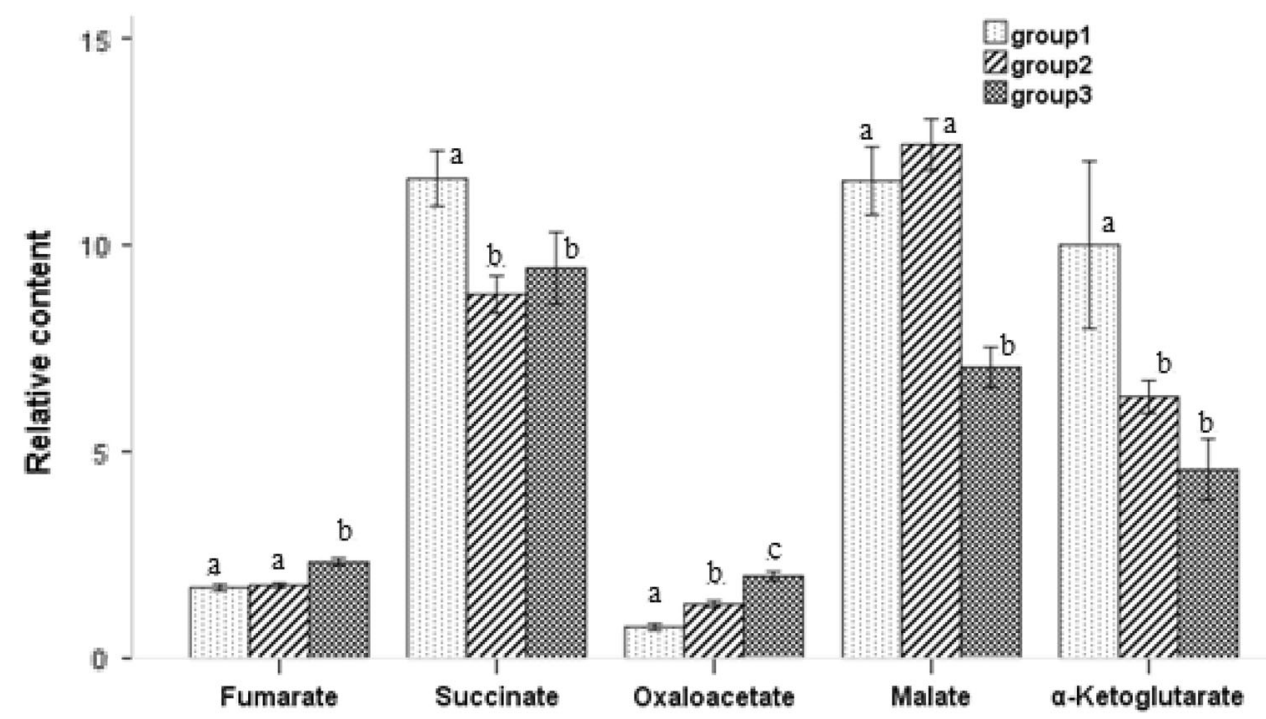

Fig. 3 Relative concentration of TCA metabolites in shrimp hepatopancreas after WSSV infected

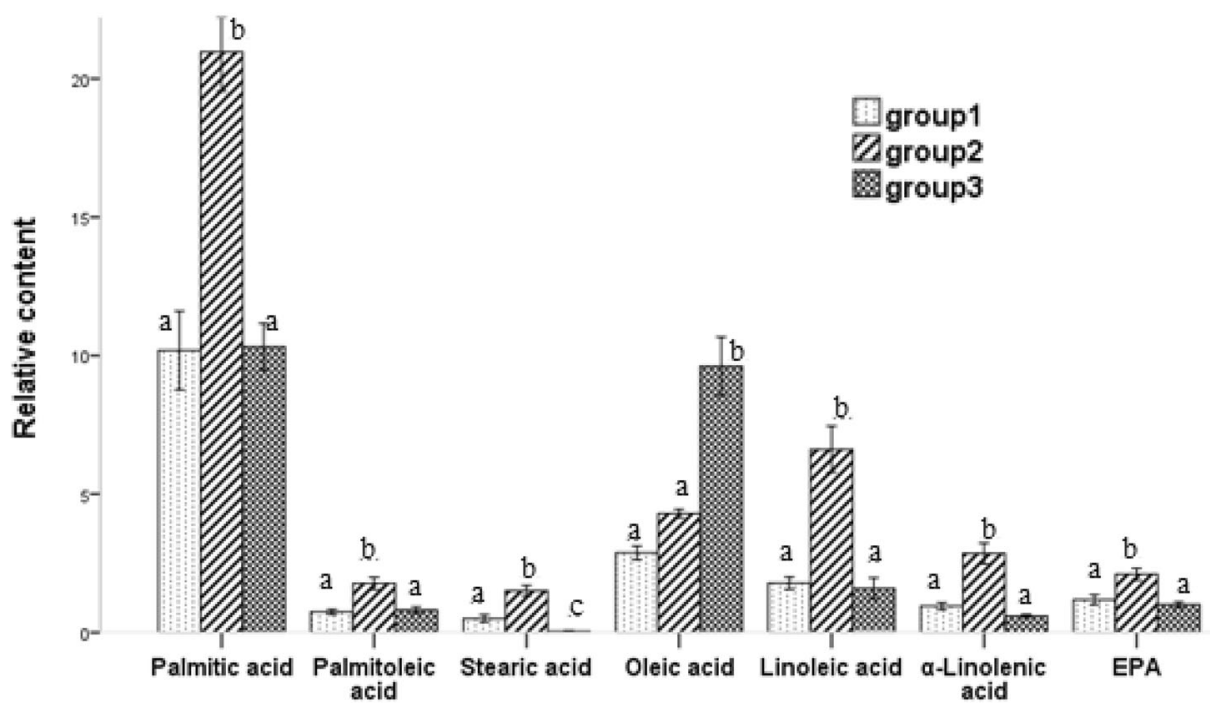

Fig. 4 Relative concentration of fatty acid metabolites in shrimp hepatopancreas after WSSV infected

2 weeks, so the virus reproduction results that quote above can explain the enhanced glycolysis phenomenon in this study. When at the stage of late viral infections, glucose was large consumption and decreased. To maintain the body energy consumption, the gluconeogenesis had intensified in hepatopancreas of shrimp, and the more lactate was consumed.

The previous study (Yoganandhan 2003) had described that the organic acid increased in hepatopancreas was a usual phenomenon, and in this study the fatty acid manifested the same action. $\alpha$-ketoglutarate was in a constant state of decline, and the downstream product succinate was equal to decrease, and also the content of malate decrease significantly on later infected stage. But the oxaloacetate of shrimp was contrarily, it increases throughout the process of WSSV infected. This may concern TCA cycle enzymes, previous research had reported that proteins were significantly upregulated or downregulated (Chai et al. 2010), and in this study the citrate synthase of shrimp may inhibited, so metabolism of citric acid cycle got disordered. The disorder of amino acid metabolism caused the significant alterations on WSSV infected shrimp that 14 amino acids were upregulation, but it's interesting when at the stage of before death, the concentration of 11 amino acids form above 14 amino acids decrease to normal level of control. An obviously, phenomenon was the content of 
Table 1 Relative concentration of amino acid metabolites in shrimp hepatopancreas of group1, group2 and group3 after WSSV infected

\begin{tabular}{|c|c|c|c|c|c|}
\hline \multirow[t]{2}{*}{ Amino acid } & \multirow[t]{2}{*}{ Group 1 (control) } & \multirow[t]{2}{*}{ Group 2 (infective stage) } & \multirow[t]{2}{*}{ Group 3 (death stage) } & \multicolumn{2}{|c|}{ Regulation direction } \\
\hline & & & & Group 2/group 1 & Group 3/Group 1 \\
\hline Gly & 51.34 & 81.95 & 106.57 & $\uparrow *$ & $\uparrow *$ \\
\hline Ala & 25.65 & 34.94 & 14.89 & $\uparrow *$ & $\downarrow^{*}$ \\
\hline Asn & 0.82 & 1.03 & 0.55 & $\uparrow *$ & $\downarrow^{*}$ \\
\hline Asp & 3.30 & 4.11 & 4.82 & - & $\uparrow *$ \\
\hline Pro & 29.99 & 29.60 & 22.22 & - & $\downarrow^{*}$ \\
\hline Glu & 5.33 & 5.18 & 3.91 & - & $\downarrow^{*}$ \\
\hline Val & 7.28 & 10.08 & 7.09 & $\uparrow *$ & - \\
\hline The & 2.32 & 2.88 & 1.97 & $\uparrow *$ & - \\
\hline Lys & 9.14 & 14.70 & 8.72 & $\uparrow *$ & - \\
\hline His & 1.48 & 2.72 & 1.39 & $\uparrow *$ & - \\
\hline Try & 1.69 & 2.81 & 1.50 & $\uparrow *$ & - \\
\hline Leu & 12.41 & 19.66 & 13.80 & $\uparrow *$ & - \\
\hline Ile & 5.01 & 6.96 & 5.25 & $\uparrow *$ & - \\
\hline Cys & 1.90 & 2.78 & 2.42 & $\uparrow *$ & - \\
\hline Arg & 6.59 & 12.99 & 7.54 & $\uparrow *$ & - \\
\hline Phe & 3.01 & 4.72 & 4.08 & $\uparrow *$ & - \\
\hline Tyr & 6.54 & 10.77 & 8.85 & $\uparrow *$ & - \\
\hline Ser & 7.88 & 8.43 & 7.24 & - & - \\
\hline Gln & 3.18 & 4.31 & 3.81 & - & - \\
\hline
\end{tabular}

The relative concentration of each metabolite is an average of data from 20 biological replicates using GCMS. The star (Asterisk) indicate significant $(p<0.05)$ difference. The short dash line indicate no significant difference. Up arrow and Down arrow represents the upregulation and downregulation respectively

inhibitory amino acid glycine sustained raise, so it's possibility the glycine was the major product of virus cell metabolism. Yoganandhan (2003) support our observation on amino acid metabolism of infected stage, but the trend of amino acid and fatty acid recovers at later stage may concerned with viral reproduction and it need more subsequently study to explain the mechanism.

The results of this study indicated that glucose metabolism and fatty acid metabolism would be abnormal, so that metrics can be used to monitor the shrimp of WSSV infected and determine the extent of the disease.

Acknowledgements This study was supported by the Shanghai Shrimp Industry Technology System Program and Laboratory Technician Team building program of china shanghai education commission (B1-5407-13-0000-5).

Open Access This article is distributed under the terms of the Creative Commons Attribution 4.0 International License (http:// creativecommons.org/licenses/by/4.0/), which permits unrestricted use, distribution, and reproduction in any medium, provided you give appropriate credit to the original author(s) and the source, provide a link to the Creative Commons license, and indicate if changes were made.

\section{References}

Bruggeman LA (2007) Viral subversion mechanisms in chronic kidney disease pathogenesis. Clin J Am Soc Nephrol 2(suppl 1):S13-S19

Chai YM, Yu SS, Zhao XF, Zhu Q, Wang JX (2010) Comparative proteomic profiles of the hepatopancreas in Fenneropenaeus chinensis response to white spot syndrome virus. Fish Shellfish Immunol 29:480-486

Chen IT et al (2011) White spot syndrome virus induces metabolic changes resembling the warburg effect in shrimp hemocytes in the early stage of infection. J Virol 85:12919-12928

Galvan-Alvarez D, Mendoza-Cano F, Hernandez-Lopez J, Sanchez-Paz A (2012) Experimental evidence of metabolic disturbance in the white shrimp Penaeus vannamei induced by the Infectious Hypodermal and Hematopoietic Necrosis Virus (IHHNV). J Invertebr Pathol 111:60-67

Heaton NS, Randall G (2011) Multifaceted roles for lipids in viral infection. Trends Microbiol 19:368-375 
Leu JH et al (2007) Comparative analysis of differentially expressed genes in normal and white spot syndrome virus infected Penaeus monodon. BMC Genomics 8:120

Leu JH, Lin SJ, Huang JY, Chen TC, Lo CF (2013) A model for apoptotic interaction between white spot syndrome virus and shrimp. Fish Shellfish Immunol 34:1011-1017

Li S, Zhang X, Sun Z, Li F, Xiang J (2013) Transcriptome analysis on Chinese shrimp Fenneropenaeus chinensis during WSSV acute infection. PLoS ONE 8:e58627

Liu PF, Liu QH, Wu Y, Jie H (2015) A pilot metabolic profiling study in hepatopancreas of Litopenaeus vannamei with white spot syndrome virus based on (1) H NMR spectroscopy. J Invertebr Pathol 124:51-56

Lo CF et al (1996) White spot syndrome baculovirus (WSBV) detected in cultured and captured shrimp, crabs and other arthropods. Dis Aquat Org 27:215-225

Sánchez-Paz A (2010) White spot syndrome virus: an overview on an emergent concern. Vet Res 41:43

Solanky KS et al (2003) Application of biofluid 1H nuclear magnetic resonance-based metabonomic techniques for the analysis of the biochemical effects of dietary isoflavones on human plasma profile. Anal Biochem 323:197-204

Wu D et al (2013) Tissue metabolic responses to salt stress in wild and cultivated barley. PLoS ONE 8:e55431

Xia J, Wishart DS (2011) Web-based inference of biological patterns, functions and pathways from metabolomic data using MetaboAnalyst. Nat Protoc 6:743-760

Yoganandhan K, Thirupathi S, Hameed ASS (2003) Biochemical, physiological and hematological changes in white spot syndrome virus-infected shrimp, penaeus indicus. Aquaculture 221:1-11 